\title{
Bisphosphonates in Patients with Glucocorticoids: Time for Implementation
}

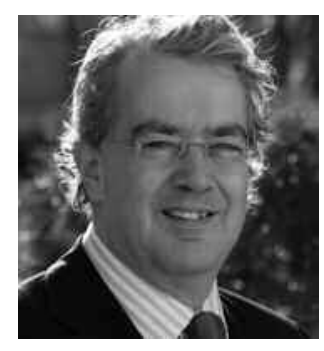

Use of glucocorticoids (GC) may have devastating effects on bone quality ${ }^{1}$. From earlier histomorphometric data, we know that bone formation is inhibited by direct suppression of osteoblast function during the use of GC, while bone resorption is unchanged or even elevated, at least partly related to decreased intestinal calcium absorption, elevated urinary calcium excretion, and a tendency to secondary hyperparathyroidism ${ }^{2,3}$. However, exciting new data provide additional insights into the pathogenesis of Glucocorticoid Induced OsteoPorosis $(\mathrm{GIOP})^{2,3}$ : (1) upregulation of the Wnt inhibitor Dickkopf-1, leading to inhibition of bone formation; (2) increased apoptosis of osteoblasts and osteocytes; (3) differentiation of mesenchymal stem cells into the direction of adipocytes, instead of into osteoblasts; (4) elevated lifespan of osteoclasts, due to decreased apoptosis; and (5) upregulation of expression of RANKL in osteoblasts (and downregulation of osteoprotegerin).

Nevertheless, it is important to realize that the (negative) direct and indirect effects of GC on bone are counteracted by the immunosuppressive effects of $\mathrm{GC}$ on the underlying disease ${ }^{2,3}$. Another complicating factor is that use of GC and the underlying disease may both be associated with muscular weakness and, subsequently, increased risk of falling.

Use of GC is associated with bone loss, particularly in the early phase of treatment, when the dosage of GC is usually high and the underlying disease for which the GC are prescribed is active. In contrast, in the chronic phase, bone loss may be mild in GC-treated patients, related to the use of lower dosages of $\mathrm{GC}$ and to low or preferably absent disease activity.

In addition, use of GC is also associated with elevated risk of fracture: In a retrospective cohort study with 244,235 GC-treated patients and the same number of age and sex matched controls, the fracture risk was elevated in GC users and was dose-related ${ }^{4}$. Because fracture risk is high in patients treated with GC, and because effective antiosteoporotic drugs, particularly bisphosphonates, are available, the use of bisphosphonates is advocated in guidelines for (elderly) patients treated with at least $7.5 \mathrm{mg}$ prednisone/day for at least 3 months ${ }^{5,6}$.

\section{SUBOPTIMAL ADHERENCE TO THERAPY IN GC USERS}

Unfortunately, implementation of these guidelines is diffi- cult. In the US, only $48 \%$ of GC-treated patients received bone mineral density (BMD) testing or medication for osteoporosis $^{7}$, and comparable data were found in The Netherlands ${ }^{8}$. It has been reported that lack of knowledge by physicians, having limited time during the outpatient-clinic visit, and patient-related factors of nonadherence are among the important barriers in the management of $\mathrm{GIOP}^{9}$. In more detail, the following patient-related factors might play a role:

1. Antiosteoporotic drugs are prescribed for prevention of future fractures. In comparable circumstances, e.g., hypertension, low adherence to therapy is also a well known and important issue, while in patients with arthritis, adherence is usually higher, because antiinflammatory drugs induce symptomatic relief of pain and inflammation.

2. Patients starting GC are usually ill and are getting informed about their underlying disease and possible side effects of GC, including hypertension, diabetes, infections, and osteoporosis. Thus, osteoporosis is just one item on a long list of possible side effects, for which it might be necessary to take 2 to 3 types of additional drugs.

3. Monitoring effects of antiosteoporotic drugs is not optimal: changes in BMD can be measured only after 2 to 3 years of therapy. An alternative is the followup of changes in bone markers, which can be observed after only 2 to 3 months of therapy, but measurements of bone markers are limited because of a relatively high analytical and biological variation, and because of the costs.

4. General phenomena not directly related to bisphosphonates:

(a) When the number of prescribed drugs is increasing, the risk of nonadherence rises; (b) antiosteoporotic drugs are usually prescribed in the elderly, in whom adherence might be limited due to early dementia; and (c) some patients have a "general negative opinion against drugs."

\section{HOW TO IMPROVE ADHERENCE TO THERAPY IN GC USERS?}

A key element in improving adherence in GIOP is the model of "shared decision-making." In contrast to the "traditional, paternalistic model," in which use of bisphosphonates is advocated "without any clear explanation," in a shared decision-making model the patient and the physician discuss the pros and cons of starting antiosteoporotic drugs. 
During this discussion, the use of the World Health Organization absolute fracture risk model (FRAX; http://www.shef.ac.uk/FRAX/) might be very useful, since it gives the patient better insight into his/her future fracture risk $^{10}$. It has been proposed to refer these patients to a "GIOP outpatient clinic," in which both physicians and nurses focus on intensive monitoring and followup of GIOP patients: patient retention of knowledge, doing exercises, and 25-OH vitamin D levels all significantly improved, while adherence to therapy was $91 \%{ }^{11}$. It is possible that these specialized clinics are a step too far, but the idea of spending more time on patient education and followup might be a crucial factor for adherence to therapy.

Another issue that might have an important effect on adherence to therapy is dosing frequency of the prescribed drugs. From data in postmenopausal women, we know that a low dosing frequency is associated with better adherence to therapy ${ }^{12}$ : a greater proportion of patients who persisted with therapy after 12 months were in the once-weekly treatment group versus the once-daily group: $52 \%$ (once-weekly) and $40 \%$ (once-daily) in the UK; data were comparable in the US (44\% vs 32\%) and in France (51\% and 44\%). In a large, retrospective study, higher adherence to bisphosphonate therapy was associated with statistically significantly fewer fractures: vertebral fractures occurred in $1.8 \%$ versus $2.8 \%$ and nonvertebral fractures in $7.1 \%$ versus $8.8 \%$, respectively, in compliant versus noncompliant patients ${ }^{13}$. The interpretation of these results is complicated: the higher fracture rate in patients with low adherence to therapy might result from inadequate adherence to therapy; but low adherence could also be related to other factors (e.g. comorbidity, lower education and/or socioeconomic levels) that in themselves may be associated with fractures.

\section{NEW DATA ON ONCE-WEEKLY ALENDRONATE IN GIOP}

In this issue of The Journal, Stoch, et al describe the effects of once-weekly alendronate $(70 \mathrm{mg}$ ) in 114 GC-treated patients versus 59 GC-treated patients who received placebo: the primary endpoint was the percentage change in BMD of the lumbar spine after 1 year ${ }^{14}$. All patients received supplemental daily calcium $(1000 \mathrm{mg})$ and $400 \mathrm{IU}$ vitamin $\mathrm{D}$. The issue of whether or not to offer placebo to GC-treated patients was elegantly resolved by excluding patients at high fracture risk based on low BMD or previous fractures. A significant treatment difference for the mean percentage change from baseline was observed for the lumbar spine (treatment difference 2.92\%; $<<0.001$ ) and total hip (treatment difference $1.19 \% ; \mathrm{p}=0.008$ ). Compliance was $88 \%$ (alendronate) and $87 \%$ (placebo).

How to interpret these data? (1) The changes in BMD are favorable at both the spine and the hips in the alendronate-treated patients. It is important to realize that the clinically relevant outcome measure in (GC-treated) patients with osteoporosis is occurrence of fractures, not change in BMD. However, it has been generally accepted that for compounds having demonstrated antifracture efficacy in postmenopausal women, an extension of the indication could be given for a new dose, based on the demonstration of changes in $\mathrm{BMD}^{15}$. (2) The alendronate-induced changes in BMD in the Stoch, et al study are comparable to data from Saag, et $a l^{16}$ (477 men and women), in which they observed an increase in BMD of the spine of $2.9 \%$, and with data from Dutch rheumatologists in 163 GC-treated patients with rheumatoid arthritis, all treated with low-dose prednisone, in which increased spine BMD of $+4.7 \%$ was found ${ }^{17}$. (3) Changes in bone markers: the urinary excretion of NTX/Cr decreased by $-32 \%$ at month 12 in the alendronate group, and $-16 \%$ in the placebo group (the latter probably related to the concomitant use of calcium and vita$\min \mathrm{D})$. The difference between the decrease in bone resorption markers is smaller than in previous studies $\left(-60 \%{ }^{16}\right.$ and $-51 \%{ }^{17}$ ). The reasons for the small treatment effect and the absence of a statistically significant difference between alendronate and placebo group in the Stoch study are unclear. Possible explanations are enrollment of relatively low-risk patients and discontinuation of patients with more than 5\% bone loss during the study. (4) Fractures were observed in 6 of the 114 alendronate-treated patients and in none of the 59 placebo patients. Since the study was not powered to detect a difference in fracture incidence, no conclusions can be based on these fracture data. (5) No statistically significant differences were observed in (gastrointestinal) side effects. Two alendronate-treated and no placebo patients died of cardiac arrest during the treatment period. Although this difference is also not statistically significant, it would be interesting to know whether atrial fibrillation might or might not have played a role in these patients. (6) Adherence to therapy is very high in both patient groups, but it is well known that compliance in randomized controlled trials is not the same as in daily practice.

\section{WHAT CAN WE LEARN FROM THIS TRIAL?}

Of greatest importance, this study highlights the favorable effects of once-weekly alendronate for the prevention of GC-induced bone loss. Since earlier studies have shown positive effects of alendronate in GC-treated patients versus placebo $^{16,17}$ and versus active vitamin D (calcitriol) ${ }^{18}$ administered once daily, and since we know that a lower dosing frequency seems to be associated with better adherence to therapy ${ }^{12}$, demonstration of favorable effects of a once-weekly regimen is very welcome. Other treatment options in GC users include risedronate, for which in a large study $(\mathrm{n}=518)$ in GC-treated patients the vertebral fracture rate was reduced by $70 \%{ }^{19}$. Remarkably, it was recently shown that a single once-yearly infusion with zoledronic acid was not inferior to and was probably even more effective than once-daily (oral) risedronate in GC-treated 
patients ${ }^{20}$. The use of the anabolic agent teriparatide might be attractive for GC-treated patients with severe osteoporosis ${ }^{21}$.

The next step is implementation. Use of bisphosphonates in combination with lifestyle measures (adequate supplementation of calcium and vitamin D, exercise therapy, and prevention of falling) in all chronically GC-treated patients who are at high risk for fractures is certainly one of the challenges for rheumatologists (and other physicians prescribing glucocorticoids) in the near future!

WILLEM F. LEMS, $\mathrm{MD}, \mathrm{PhD}$,

Rheumatologist,

Department of Rheumatology,

VU University Medical Center,

3A 64 Postbox 7057,

1007 MB, Amsterdam, The Netherlands

Address correspondence to Prof.Lems.E-mail: wf.lems@vumc.nl

\section{REFERENCES}

1. Lems WF. Bisphosphonates and glucocorticoids: effects on bone quality. Arthritis Rheum 2007;56:3518-22.

2. van Staa TP. The pathogenesis, epidemiology and management of glucocorticoid-induced osteoporosis. Calcif Tissue Int 2006;79:129-37.

3. Curtis JR, Saag KG. Prevention and treatment of glucocorticoid-induced osteoporosis. Curr Osteoporos Rep 2007;5:14-21.

4. van Staa TP, Leufkens HG, Abenhaim L, Zhang B, Cooper C. Oral corticosteroids and fracture risk: relationship to daily and cumulative doses. Rheumatology 2000;39:1383-9.

5. Recommendations for the prevention and treatment of glucocorticoid-induced osteoporosis: 2001 update. American College of Rheumatology Adhoc Committee on Glucocorticoid-induced Osteoporosis. Arthritis Rheum 2001;44:1496-503.

6. Geusens PP, De Nijs RN, Lems WF, et al. Prevention of glucocorticoid osteoporosis: a consensus document of the Dutch Society for Rheumatology. Ann Rheum Dis 2004;63:324-5.

7. Solomon DH, Katz JN, Cabral D, et al. Osteoporosis management in patients with rheumatoid arthritis: evidence for improvement. Arthritis Rheum 2006;55:873-7.

8. Duyvendak M, Naunton M, Atthobari J, et al. Corticosteroid-induced osteoporosis prevention: Longitudinal practice patterns in the Netherlands 2001-2005. Osteoporos Int 2007;18:1429-33.
9. Guzman-Clark JR, Fang MA, Sehl ME, et al. Barriers in the management of glucocorticoid-induced osteoporosis. Arthritis Rheum 2007;57:140-6.

10. Dawson Hughes B, Tosteson ANA, Melton LJ III, et al. Implications of absolute fracture risk assessment for osteoporosis guidelines in the USA. Osteoporos Int 2008;19:449-58.

11. Newman ED, Matzko CK, Olenginski TP, et al. Glucocorticoid-Induced Osteoporosis Program: a novel, comprehensive and highly successful program with improved outcomes at 1 year. Osteoporos Int 2006;17:1428-34.

12. Cramer JA, Lynch NO, Gaudin AF, Walker M, Cowell W. The effect of dosing frequency on compliance and persistence with bisphosphonate therapy in postmenopausal women: a comparison of studies in the United States, the United Kingdom, and France. Clin Ther 2006;28:1686-94.

13. Siris ES, Harris ST, Rosen CJ, et al. Adherence to bisphosphonate therapy and fracture rates in osteoporotic women: relationship to vertebral and nonvertebral fractures from 2 US claims databases. Mayo Clin Proc 2008;81:1013-22.

14. Stoch SA, Saag KG, Greenwald M, et al. Once-weekly oral alendronate $70 \mathrm{mg}$ in patients with glucocorticoid-induced bone loss: a randomized, placebo-controlled trial: J Rheumatol 2009;36:1705-14.

15. European Medicines Agency. Guideline on the evaluation of new medical products in the treatment of primary osteoporosis. London, 14 December 2005.

16. Saag K, Emkey R, Schnitzer TJ, al. Alendronate for the prevention and treatment of glucocorticoid-induced osteoporosis. N Engl J Med 1998;339:292-9.

17. Lems WF, Lodder MC, Lips P, et al. Positive effect of alendronate on bone mineral density and markers of bone turnover in patients with rheumatoid arthritis on chronic treatment with low-dose prednisone. Osteoporos Int 2006;17:716-23.

18. De Nijs RN, Jacobs JW, Lems WF, et al. Alendronate or alfacalcidol in glucocorticoid-induced osteoporosis. N Engl J Med 2006;355:675-84.

19. Wallach S, Cohen S, Reid DM, et al. Effects of risedronate treatment on bone density and vertebral fracture in patients on corticosteroid therapy. Calcif Tissue Int 2000;67:277-85.

20. Reid DM, Devogelaer JP, Saag K, et al. Zoledronic acid and risedronate in the prevention and treatment of GIOP. Lancet 2009;373:1253-63.

21. Saag KG, Shane E, Boonen S, et al. Teriparatide or alendronate in glucocorticoid-induced osteoporosis. N Engl J Med 2007;357:2028-39.

J Rheumatol 2009;36:1570-2; doi:10.3899/jrheum.090535 